\title{
Optimizing the Recognition of Surface Crystallography
}

\author{
Luděk Frank, Filip Mika and Ilona Müllerová
}

Institute of Scientific Instruments of the ASCR, v. v. i., Královopolská 147, 61264 Brno, Czech Republic

Crystallographic information about solid samples acquired at high lateral resolution, along with information about electronic structure and properties, are crucial characteristics in the development and diagnostics of materials. In the nanotechnology era, surface-bound structures are of increasing importance and the same is true of approaches enabling the enhanced surface sensitivity of methods providing crystallographic data. Should high lateral resolution be combined with high surface sensitivity, the application of backscattered electrons (BSE) is both a traditional and highly prospective solution with an information depth shortening with the decreasing energy of electrons. Low Energy Electron Diffraction (LEED) performed at units or tens of eV, preferably in the direct imaging (LEEM) or scanned (SLEEM) microscopic modes, is an ultimate method. These methods provide complex local information about the crystal system and its spatial orientation, including influences from electron/electron interaction, for which reason dynamical LEED theory is required for interpretation. These methods are needlessly complicated for routine analyses of materials and provide superfluous information. The Electron BackScatter Diffraction (EBSD) method, based on the acquisition of diffraction patterns with a camera and analysis of Kikuchi bands, is available at electron energies in tens of $\mathrm{keV}$ (less frequently between 5 and $10 \mathrm{keV}$ ) [1]. EBSD suffers from rather slow data acquisition and processing and from lateral resolution reduced by the high tilt of the sample. On the other hand, the method is well established and supported by decades of development of software for experiment control and data processing algorithms. However, some modern materials such as ultrafine grained metals have begun to go beyond the scope of the capabilities of EBSD when information is needed about the size and shape distributions of nanometer-sized grains over large areas. In these cases, it would be sufficient to obtain high contrast between crystal grains using an imaging mode with good surface sensitivity, high lateral resolution and fast data acquisition. The aim of this paper is to examine the ability of BSE emission to provide high contrast of crystal grains, and to optimize the BSE mode in the SEM for this purpose where only a standard one-channel BSE detector is available. We will restrict ourselves to units of $\mathrm{keV}$, i.e. to the low limit of the channeling contrast range.

The conditions defining the present study have been determined as follows:

- a polycrystalline metal sample, cleaned in situ with an ion beam and reconstructed by heating in an ultrahigh vacuum device in order to acquire an atomically clean surface free of damaged overlayers

- a scanned electron beam of an energy between 2 and $8 \mathrm{keV}$

- a sample situated in a field-free space

- the acquisition of elastic as well as inelastic BSE with a detector without front bias

- a detector with a narrow acceptance angle, movable in one azimuthal plane above the sample surface

- measurement of the planar grain contrast in several couples of grains in dependence on the polar angle of emission and the energy of incident electrons

Experiments were performed in an ultrahigh vacuum SLEEM device developed in the author's laboratory [2]. The device (Figure 1) is equipped with a pure electrostatic illumination column, detectors 
of transmitted, backscattered and side-scattered electrons as well as Auger electrons, and facilities appropriate as surface analysis apparatus including in situ cleaning with an ion beam, specimen heating to $1,000{ }^{\circ} \mathrm{C}$, sample manipulations controlled from outside the vacuum, etc. No electric field surrounded the sample. The beam energy was set between 2 and $8 \mathrm{keV}$ with a current from 0.3 to $1.5 \mathrm{nA}$. The detector used for the study was a scintillator based detector with an active area of $7 \mathrm{~mm}$ in diameter that was moved through 37 positions where it accepted the polar and azimuthal angular ranges shown in Figure 2. The spatial angle of acceptance varies up to a maximum of $0.17 \mathrm{sr}$ for a polar angle of $45^{\circ}$. Obviously, the acceptance cones, chosen in order to secure a sufficient signal-to-noise ratio, are too wide to resolve details in the BSE distribution on the level of various kinds of Kikuchi bands. Only contrast relations based on envelopes of the BSE distribution from crystals grains were addressed.
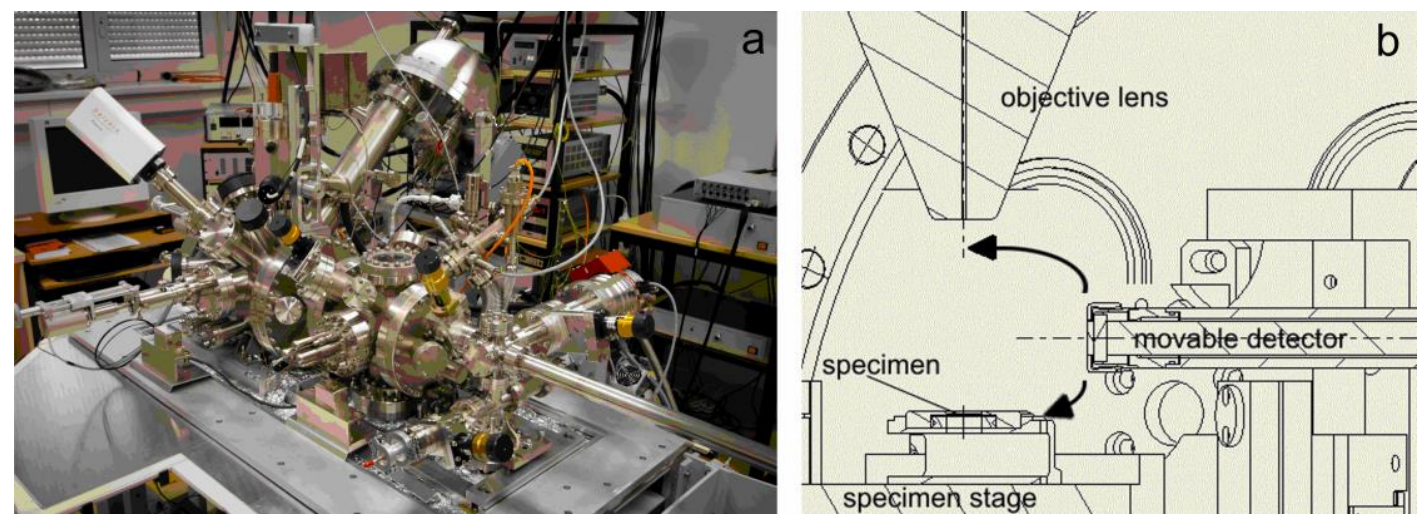

Figure 1. The UHV SLEEM apparatus with analytic, preparation and loading chambers (a); crosssection of the neighborhood of the sample showing the detector of side-scattered electrons (b).
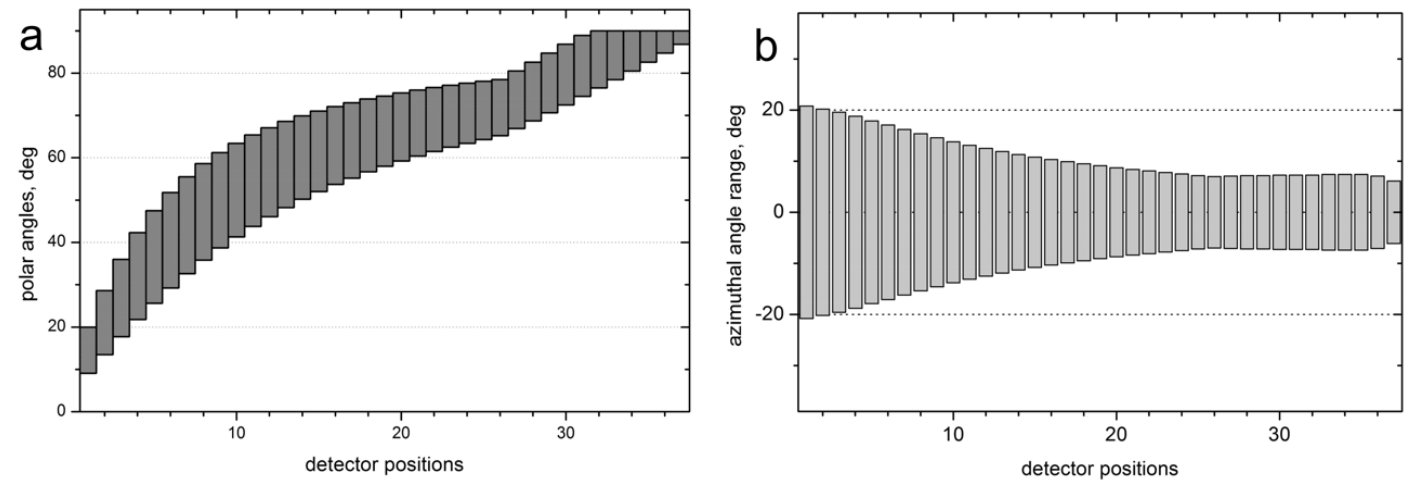

Figure 2. Ranges of the azimuthal and polar angles of the BSE emission incident on the detector.

As the surface sensitivity, i.e. the information depth of imaging, is one of the key parameters of the study, we have used electron energies as low as $2 \mathrm{keV}$, i.e. a low limit for detection without any bias applied to the scintillation detector that would deform the signal trajectories. Furthermore, we have used a sample cleaned and surface reconstructed in situ and therefore free of any amorphous surface layers. As a representative material from the middle of the periodic table, polycrystalline copper was used with a small content of tin (1.7\% according to EDS analysis) (Figure 3). The Auger electron spectrum in Figure 3 shows the absence of oxygen as evidence of surface cleanliness, though it also shows the presence of a little carbon of unknown origin. We have chosen four couples of neighboring grains in a field of view of about $350 \mu \mathrm{m}$ in diameter for contrast measurement (Figure 4). Their orientations were 
established by means of EBSD - see Figure 5. The grain couples were chosen randomly from those exhibiting non-negligible mutual contrast through the whole energy range of 2 to $8 \mathrm{keV}$.

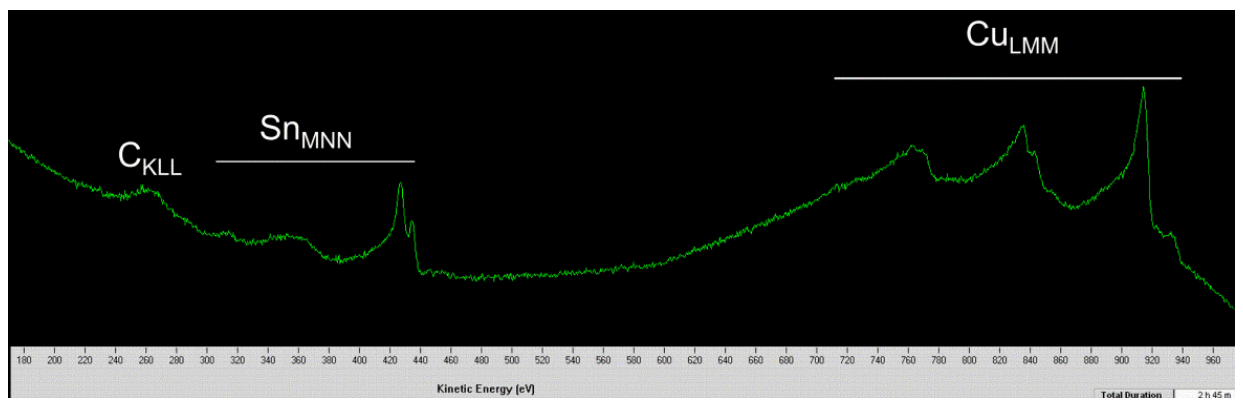

Figure 3. Spectrum of Auger electrons taken from the field of view shown in Figure 4.

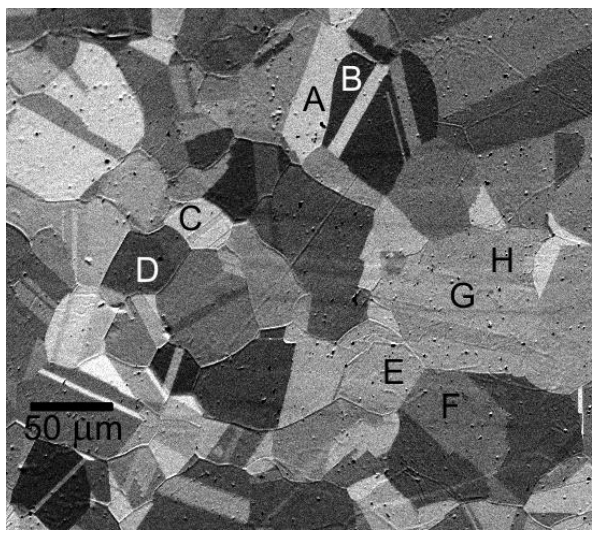

Figure 4. BSE micrograph taken at $8 \mathrm{keV}$, showing the grains chosen for contrast measurements.

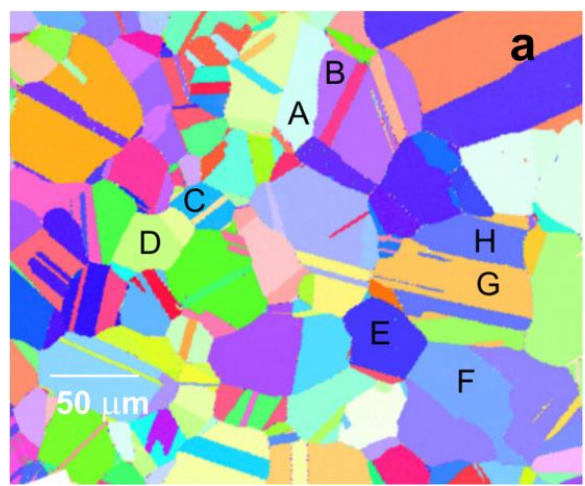

\begin{tabular}{|c|c|c|}
\hline Grain & $\{h k I\}$ & $<u v w>b$ \\
\hline A & $\left\{\begin{array}{lll}15 & 6 & 23\end{array}\right\}$ & $<21 \quad 5-15>$ \\
\hline B & $\left\{\begin{array}{lll}-11 & -15 & 25\end{array}\right\}$ & $<1015>$ \\
\hline c & $\left\{\begin{array}{lll}9 & 5 & 9\end{array}\right\}$ & $<18-9-13>$ \\
\hline D & $\left\{\begin{array}{lll}-6 & -1 & 10\end{array}\right\}$ & $<\begin{array}{lll}7 & -2 & 4\end{array}>$ \\
\hline E & $\left\{\begin{array}{ll}12-13 & 15\end{array}\right\}$ & $<8-3-9\rangle$ \\
\hline $\mathbf{F}$ & $\left\{\begin{array}{lll}-17-11 & 21\end{array}\right\}$ & $\begin{array}{lll}21 & 0 & 17\end{array}>$ \\
\hline G & $\left\{\begin{array}{lll}-1 & -7 & 20\end{array}\right\}$ & $<1932>$ \\
\hline H & $\left\{\begin{array}{lll}-14 & 10 & 17\end{array}\right\}$ & $<19-418>$ \\
\hline
\end{tabular}

Figure 5. An EBSD orientation map of the field of view selected (a), orientation of the labeled grains (the $\langle\mathrm{u} \mathrm{v} \mathrm{w}>$ vector is in the azimuthal plane of detector movement) (b).

The series of measurements consisted of the acquisition of datacubes of micrographs with the detector position as the third dimension. Image signals were averaged off-line within circles centered in the grains and contrasts were calculated as

$$
\text { contrast } X / Y=\frac{\bar{X}}{\bar{Y}}\left[1+\frac{\sigma^{2}(Y)}{\bar{Y}^{2}}\right]
$$

where $\bar{X}, \bar{Y}$ are signal averages inside grains. The results are plotted in Figure 6 with respect to the polar 
angle of the detector axis.
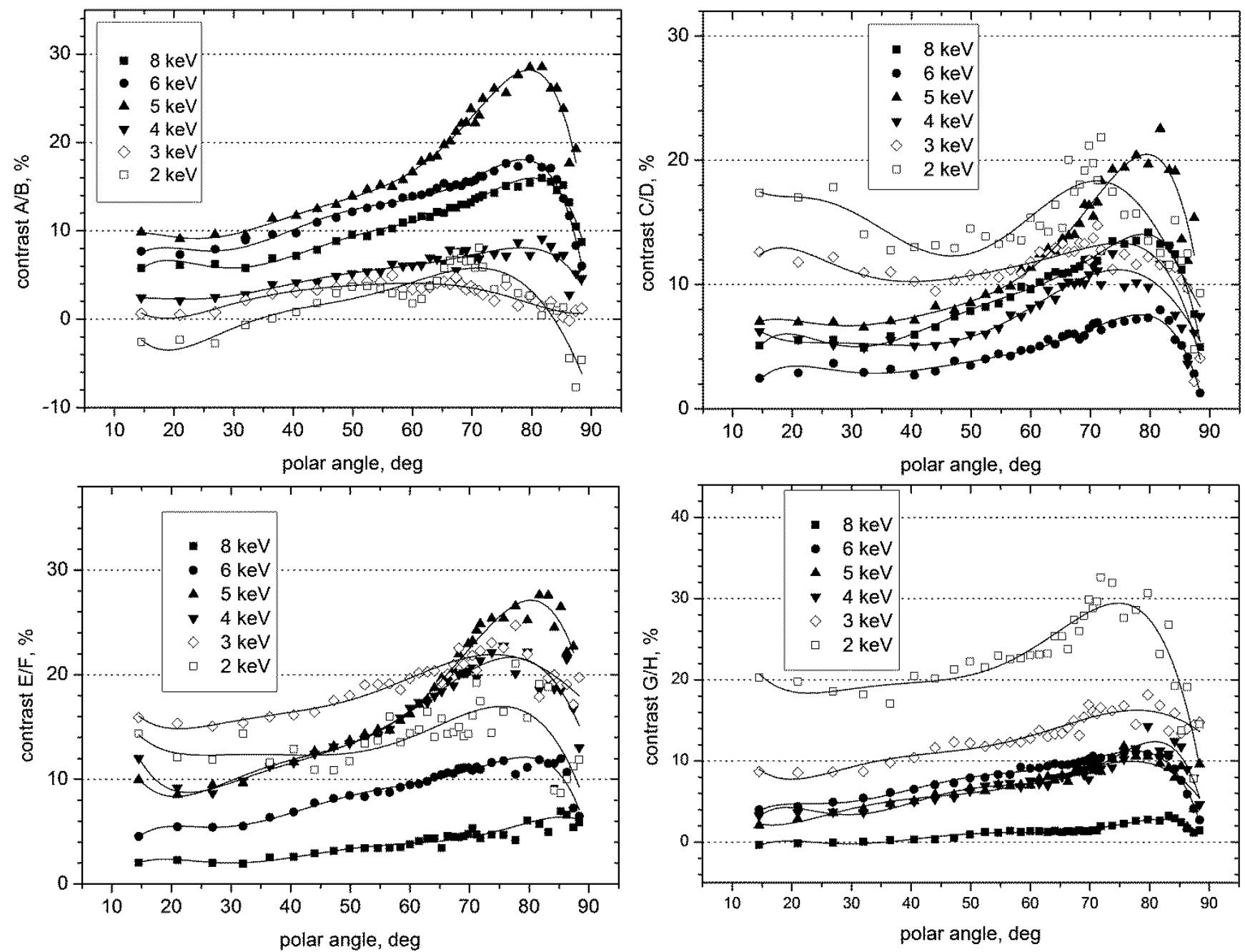

Figure 6. Contrasts of four couples of grains shown in Figures 4 and 5, plotted versus polar angle of the detector axis for various primary energies of electrons.

Across the full scale of polar angles, the appearance of the BSE micrograph varies not only in contrast but also in the signal-to-noise ratio (SNR) dependent on the angular distribution of the BSE emission and on the varying solid acceptance angle of the detector (sees Figure 2). Other contrasts usually observed connected with various surface overlayers are suppressed due to thorough cleaning of the surface, but the topographic contrast remains with its proportion enhancing with an increasing polar angle (Figure 7).

At first sight Figure 6 indicates a general property of the grain contrast curves, namely their growth up to a maximum at high polar angles not far from the tangential takeoff. The measured data points were fitted with sixth order polynomials and we have plotted the positions and heights of the maxima of fitting curves in Figure 8. With two exceptions, maximum grain contrast is obtained between polar angles of $70^{\circ}$ and $82^{\circ}$ independently of the orientations of the grains and the energy of electrons. On the other hand, the heights of the contrast maxima do not obey particularly clear rules. For some grain 
couples the contrast increases slightly with the decreasing energy of electrons, but the opposite trend also appears and no dependence is monotone.
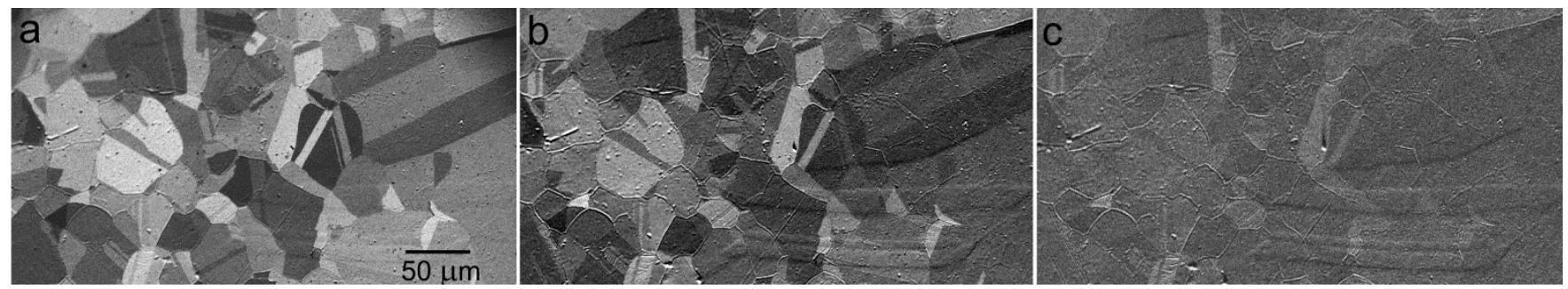

Figure 7. Samples from the series of micrographs taken at $8 \mathrm{keV}$ : (a) maximum SNR for the polar angle range $25.6^{\circ}$ to $47.5^{\circ}$, (b) maximum contrast for the range $72.5^{\circ}$ to $86.8^{\circ}$, (c) maximum topographic contrast for the range $86.8^{\circ}$ to $90^{\circ}$.
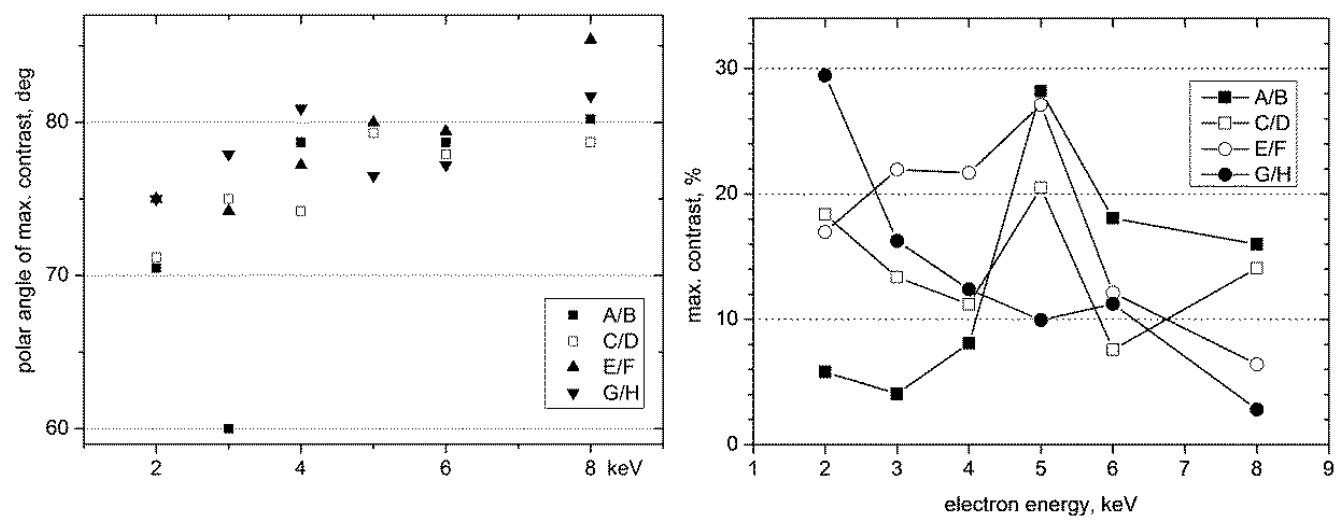

Figure 8. Position and height of the maxima of polynomial curves fitted to the grain contrast data.

We simulated the scattering of 2 and $8 \mathrm{keV}$ electrons in pure copper using software published by Kieft and Bosch in an attempt to interpret the observed relations [3]. This software considers the sample material as amorphous, so it produces results averaged over all crystal orientations. Three characteristics were extracted from data obtained when simulating $100 \mathrm{k}$ of incident electrons, namely the maximum penetration depth of an electron that was later emitted as a BSE, the number of interaction events survived by this electron inside the solid, and the distance of the point of emission of this electron from the point of its impact on the surface. The parameters were then sorted according to the polar angle of the BSE emission from the surface and grouped into classes $10^{\circ}$ wide. In each class, the median was taken for the penetration depth and for the distance of the emission point, while the number of interactions was averaged within each class. The results of these simulations are shown in Figure 9.

The common maximum of the grain contrasts appearing at high polar angles can be interpreted as an effect based on the length of trajectory of a BSE inside the sample. Having an information depth three or four orders of magnitude shallower than the typical size of grains, we do not consider electrons penetrating below grains visible on the surface. Thus, if we characterize a grain by "channeling efficiency", i.e. a loss per unit length of the number of electrons capable of being backscattered, we get a grain contrast directly proportional to the length of trajectory. In Figure 9, we see that the penetration depth increases about two times between the lowest and highest polar angle, while the distance of the emission point from the impact point increases by tens of percent. In view of the rather large numbers of interactions we cannot establish true lengths of trajectory, but certainly the shortest possible is always a 
V-shape one between the surface and penetration depth levels. Along the polar angle scale, a pathlength estimated in this way extends from $4.6 \mathrm{~nm}$ to $10 \mathrm{~nm}$ for $2 \mathrm{keV}$ electrons and from $54 \mathrm{~nm}$ to $92 \mathrm{~nm}$ at 8 $\mathrm{keV}$, which roughly fits our data. With this approach we also implicitly account for the crystallographic dependence of penetration depth. Achievement of a maximum and a following break of the plot downwards can be ascribed to the oblique passage of BSE through the closest subsurface layer. Even when oxide and contamination layers, as well as a layer amorphized by the ion beam bombardment, are completely removed, multilayer relaxation and reconstruction of the surface facilitated by heating would produce a layer of a structure different from that of the bulk and tending to reduce differences in the scattering power of different crystal orientations. Naturally, with the decreasing energy of electrons, the full process concentrates toward the surface and the grain contrast maximum shifts toward lower polar angles. The absence of a fundamental relationship concerning the dependence of the grain contrast on the energy of electrons might be simply imputed to diversity in grain orientations. Anizotropy in backscattering, when solved via Bloch-wave fields, achieves units or even tens of percent with finely undulating angular distribution varying with electron energy [4] so we can obtain very changeable results when combining distributions from two crystals.
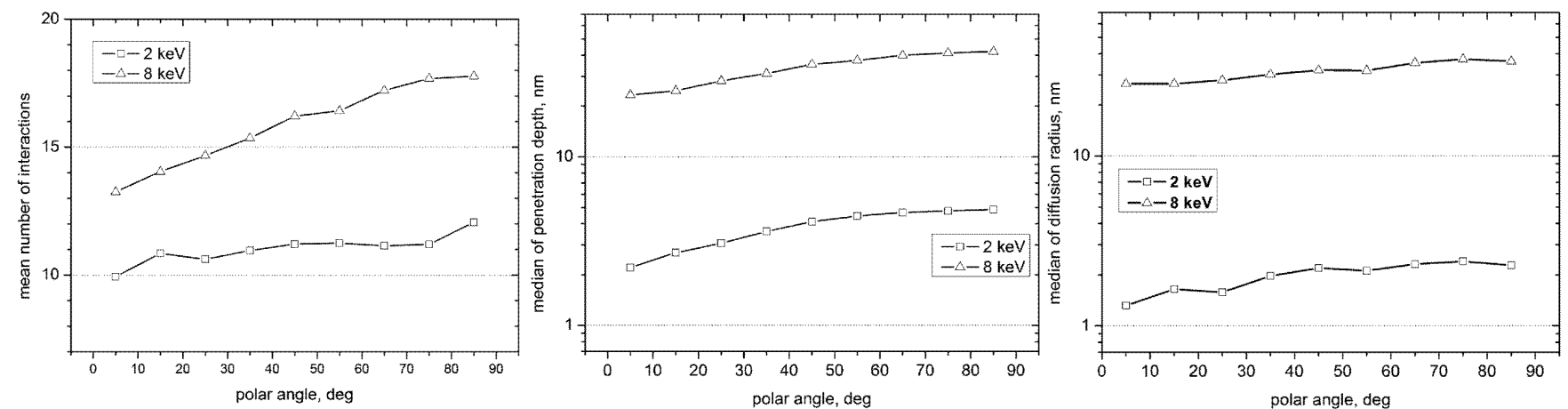

Figure 9. Simulated parameters of BSE generation in pure amorphous copper.

When applying the results of this study to routine BSE imaging of polycrystals in a standard SEM we have to take into account the presence of various adlayers on grain surfaces that make the above mentioned "subsurface" region thicker and that should therefore lower the polar angle for maximum grain contrast. Moreover, the adlayers can produce additional contrasts like those of thickness differences because of variations in reactivity. In any case, in order to optimize the crystallographic contrast we have to acquire BSE emitted at high polar angles and to tailor the landing energy of electrons to a particular structure. [5]

\section{References:}

[1] JA Venables and CJ Harland, Phil. Mag. 27 (1973), p. 1193.

[2] I Müllerová and L Frank, Adv. Imaging and Electron Phys. 128 (2003), p. 309.

[3] E Kieft and E Bosch, J. Phys. D - Appl. Phys. 41 (2008), no. 215310.

[4] L Reimer in "Scanning electron microscopy, physics of image formation and microanalysis", (Springer, Berlin) Chapt. 9.

[5] Study supported by the project TE01020118 of the TACR and by MEYS CR (LO1212). Thanks are due to Mr. Jiří Sýkora for his assistance in experiments. 